\title{
Metacognitive Invariants as Psychological-Pedagogical Factors of Training
}

\author{
Alexander G. Gein ${ }^{1}$, Vladimir P. Nekrasov ${ }^{2, *}$ \\ ${ }^{1}$ Ural Federal University, Russia \\ ${ }^{2}$ Ural Technical Institute of Communications and Informatics, Russia \\ Corresponding Author: nvp1947@mail.ru
}

Copyright (C) 2013 Horizon Research Publishing All rights reserved.

\begin{abstract}
In this paper, we propose an approach to acquire by students the cognitive competencies. This approach is based on the inclusion of metacognitive invariants in a methodical system. It is verified experimentally that the quality of the learning increases if the proposed invariants are included into academic courses.
\end{abstract}

Keywords Metacognitive Invariant, Meta-subject Skill, Concept Link, Academic Course Model Topology

\section{Introduction}

Nowadays the term competency is widely used in everything that is said and written about education and teaching. The competence approach has been introduced by J. Raven [1] having proposed 37 competences. Later the Council of Europe defined five sets of key competencies to which it attached particular importance. One of them is "the ability to study all life as the basis of discontinued education within the context of both professional and social life" [2]. It is clearly seen that the cognitive component is recognized in the formulation of this competence. In general the competences were featured as the capacity of graduates to identify problems independently and to find solutions to them based on the social and natural sciences.

It should be noticed that acquisition and mastery of cognitive competences have been considered in a number of researches. Meanwhile, they are focused on the development of the aptitude to solve productive and creative problems within one subject, frequently, within one didactic unit of the subject. This 'mechanism' can be described as a local level of the acquired cognitive competences i.e. the aptitude to apply the acquired knowledge is limited to the neighborhood of material being studied at the moment. In the practice of educational research concepts are introduced which emphasize a local nature of competences (e.g. [3]).

However, as stated above the cognitive competences of the graduate are not local. Thus, meta-subject knowledge and skills are becoming decisive in the competences acquisition.
Modern pedagogies refer to a meta-subject the knowledge and skills that are acquired in the study of different subjects, but having common distinctive features and providing generalized action techniques for solving different kinds of problems. The meta-subject skills are released in the students' capacity to find a common character in different problems, in the unified schemes and considerations, in a justified transfer of the features of one object onto another, in an extrapolation by analogy, etc.

J. Piaget [4] has singled out two basic structures which play a major role in the formation of cognitive structures in preschool and primary school children. These are classification and seriation. Operation of classification involves finding of a common trait by which objects from a diverse set can be selected and combined into one group. The seriation, vice versa, is referred as arrangement of the objects into certain order under the common feature. Further on an individual acquires such cognitive concepts as generalization, abstraction, analysis and synthesis. They are clear to be meta-subject skills. The boundary between psychology and pedagogies here lies in that psychology studies how the operations are released in an individual psyche (comprising procedures of diagnostics and measurements), rather pedagogies tries to find out a way to develop an individual's ability to effect the operations. In order to build a pedagogical model of meta-subject knowledge and skills one needs to characterize its structure. We define it as concept links, i.e. something that could be expressed as invariant when we consider different concepts, approaches, methods of solving problems, etc. The idea has something in common with J. Piaget's idea, although its nature is more common, as it is referred to more general logical universal actions rather than classification and seriation.

The purpose of this paper is to consider the logical invariants and the possibility of their use in training.

\section{Basic Concepts and Methods}

The concept of invariant as a model construction facility 
was first mentioned in the works of D.Hilbert, H.Veyl, F. Klein, H. Poincare and other mathematicians at the turn of the XX century. Namely F. Klein has formulated a fundamental idea to describe different geometries as invariants of one or other space transformation groups. Later the theory of invariants became the basis of quantum physics. In our opinion the idea of invariant can be applied effectively in discussing cognitive structures.

As stated above an invariant describes the characteristics of immutable when performing a particular group of actions. Within the context of our research we are dealing with universal logic operations implemented by students during a cognitive process. That is why we propose name metacognitive invariants for such invariants.

In [5] our initial research is summarized. We have presented the following metacognitive invariants: isomorphism, concept submission, language presentation, inheritance, homomorphism, topological nodes (source and stock), and variability of concept presentation.

Further we provide definitions and illustrate the effect and cognitive role of the invariants by the examples from different fields of knowledge. One should mention one linguistic feature of the names chosen for metacognitive invariants: classification, seriation, isomorphism, inheritance, and etc. On the one hand, they imply cognitive actions implemented by the student while working with the studied objects: classification is made, seriation is effected, isomorphism is found, inheritance determined, and etc. On the other, they generalize the aim and outcome of the actions. The research of the corresponding mechanism falls into the field of psychology. In this paper we discuss the contents of the invariants (i.e. the aim component) in the view the methodical system.

1. Isomorphism. The concept A is isomorphic towards the concept $\mathrm{B}$ if the components of both of them possess one-to-one correspondence retaining structural links.

Isomorphism is one of the strongest metacognitive invariants. On the one hand it causes the students to see which features of the objects or which factors of the process are substantial. On the other, the understanding of the isomorphism of different objects enables them to choose the most convenient form of the object to perform actions on them.

For example, in discrete mathematics the most striking evidence of this metacognitive invariant is isomorphism between theoretical-sets and logical operations. Physics operates with isomorphism between oscillation processes in mechanics (pendulum) and electro-magnetism (oscillatory circuit).

Here is another example of a task that was used in the experiment with the students ofmathematic and economic specializations.

Task 1. Find if there is isomorphism between operations of variable value assignment (programming) and inflation/deflation processes (finance).

The essence of operation assignment is that different values can be assigned to a variable with the same name wherein the previous value is lost.

The process of inflation / deflation is seen in fact that a banknote with the same face value (i.e. the 'name') is changing its purchasing power, having lost the previous one.

Moreover, the isomorphism is manifested not only in the framework of this pair of two-component systems (variable's name and variable's value), but when they are used in external processes. The overflow effect (the variable value being too large) and 'computer zero' (the variable value being too small) are well-known in computer programming. Both lead to the collapse of the computational procedure. The financial sphere suffers the same consequences - both ultrahigh and ultralow purchasing power can cause economic disaster.

In [6] it has been shown that the mastery of isomorphism as metacognitive invariant plays an important role for the development of professional skills in the field of law.It can be assumed that social and economic specialists with the underdeveloped cognitive structure make mistakes often in the assessment of the possibility of transferring solutions from one situation to another.

2. Language presentation.Concept $\mathrm{B}$ is said to be included in the language of a representation of the concept Aif a definition of the concept $\mathrm{A}$ is feasible with the use of the concept of B.Note that both concepts can be independent objects.

For example, the concept 'graph' is the language for presentation of the concept 'binary relation'. The concept 'table' is the language for presentation of the concept 'n-relation'. Meanwhile, both concepts of the 'graph' and 'table' are independent objects, they are characterized by their own features and applications independent on the concept 'relation'.

The significance of the metacognitive invariant 'language presentation' is characterized by the following example.

Task 2. An assembly line is designed for manufacturing of an apparatus. The apparatus consists of two units. The automatic machine needs three seconds (s) to connect them. To manufacture the first unit employing circuits $\mathrm{C} 1$ and $\mathrm{C} 2$ takes $5 \mathrm{~s}$, the second unit employing circuits $\mathrm{C} 2$ and $\mathrm{C} 3$ takes 6s to be manufactured. The circuit $\mathrm{C} 1$ is built out of components D1 and D2, to manufacture $\mathrm{C} 1$ need $4 \mathrm{~s}$; the circuit $\mathrm{C} 2$ employs components D2 and D3 and need 3s for manufacturing; the circuit $\mathrm{C} 3$ employs one component $\mathrm{D} 3$, it takes $2 \mathrm{~s}$ to manufacture. The manufacturing of components D1, D2 and D3 is independent on each other and can start simultaneously. The component D1 manufacturing takes $7 \mathrm{~s}$, D2 manufacturing takes 8s, D3 manufacturing takes 9s. The assembly of each circuit can commence as soon as the components needed are manufactured, the assembly of each unit can commence if necessary circuit have been made. What is the shortest time scheduled to manufacture the apparatus?

The first step in solving the problem is based on the metacognitive invariant - to select the language presentation. The situation may turn out to be at a deadlock if the student fails to realize what to begin with pondering over the 
problem. The extent of the student's independence to identify the language presentation demonstrates his level of the cognitive competence development. The first proposal is to form a table out of the data such as table 1. This is a standard information curtailment technique.

Table 1. Submission of the problem data

\begin{tabular}{|c|c|c|c|c|c|c|c|c|c|}
\hline \multirow{2}{*}{ Object } & \multicolumn{3}{|c|}{ Components } & \multicolumn{3}{c|}{ Circuits } & \multicolumn{2}{c|}{ Units } & \multirow{2}{*}{ Apparatus } \\
\cline { 2 - 9 } & D1 & D2 & D3 & C1 & C2 & C3 & U1 & U2 & \\
\hline $\begin{array}{c}\text { Time of } \\
\text { manufacturing }\end{array}$ & 7 & 8 & 9 & 4 & 3 & 2 & 5 & 6 & 3 \\
\hline
\end{tabular}

It is hoped that the analysis of the result will lead students to the conclusion that selected language does not transfer sufficient information to reflect links between components and circuits, between circuits and units. The skill to find the selected language presentation insufficiency reflects a certain level of the cognitive competence. It is hoped that the analysis of the result will lead students to the conclusion that selected language does not transfer sufficient information to reflect links between components and circuits, between circuits and units. It is possible that the students will offer the idea to portray by the edges of the graph the relationship between the components. The graph's edges have to be oriented to display the information on the sequence performance of works. The appropriate presentation is displayed in Fig 1.

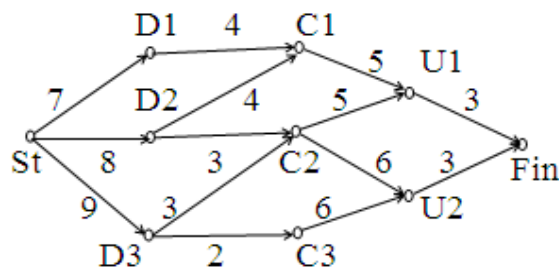

Figure 1.Submission of data relationships

Since all the works should be fulfilled the shortest time scheduled to manufacture the apparatus is defined as the longest way from the start (St) to the finish (Fin).It is not particular difficulty to solve the problem in this language of representation.

It should be noted, that the students have to find the language of presentation, i.e. a cognitive component of the problem. The search technique of the longest way is certain to be explained separately.

Since the level of the students' cognitive competence is different, a teacher has to hint at one or other techniques for solving the problem. Meanwhile, a pedagogical experiment within the course of 'Discrete Mathematics' for the IT-students has revealed that a few students (about $8 \%$ ) are able to solve the problem independently should they realize the need to select the language of presentation.

The final step of the reflexion is the most important. The students formulate themselves what kinds of problem they have learned to solve and what the solution technique implies. The level of the generalization reflexion may be different - from the formulation of the planning problems class to common understanding that methods of graph theory are useful when the problem deals with the sequence of the objects transformation into other ones. If a student understands that methods of the theory of graphs are useful dealing with the situation transformation he appreciates these as node methods and he is prepared to apply them in different situations (in non-typical ones too). The understanding is the results from the reflexion of knowledge and skills have been acquired during the process of solving one problem or another.

3. Inheritance. The concept A is an object-inheritor of the concept $\mathrm{B}$ if the concept $\mathrm{A}$ is unable to be presented by means without the concept $\mathrm{B}$.

For example, such concepts as 'binary relation', 'n-relation' and 'graph' are object-inheritor of the concept 'set'.

The concept of inheritance plays an important part in the object paradigm, e.g., in theory of computer programming. We view inheritance as a cognitive structure enabling to analyze adequately a relationship between objects and sub-objects within the system. So, the students' acquisition of this metacognitive invariant is a part of the system approach as a meta-subject skill.

4. Topological node.This concept relate to problem solving techniques. Considered are two types of topological nodes: a node-source and a node-stock.

The node-source implies the same technique or principle to be applied for solving different kinds of problems. A well-known example is to apply a certain integral concept for determining an area of the shape, a length of the curve, a mass of the body, and etc.

The node-stock is to apply different techniques for solving the same problem. The mood of thinking to find various ways to solve of one problem is an important cognitive capacity of any specialist.

G. Polya emphasizes the importance of these two types of cognitive structures to be acquired by the students in his well-known book [7].

5. Concept presentation variability. Variability of the concept presentation manifests itself in the following aspects:

a) in a concept definition;

b) in a concept content evaluation.

In a narrow sense, the variability representation of concepts can be understood as an availability of different logically equivalent definitions. Within the course of math analysis, e.g. the definition of the limit of a function in the $\varepsilon-\delta$ language (underCauchy) and in the language of sequences' convergence (under Heine) are equivalent. As a rule, the Cauchy's definition is applied to prove the number given to be the limit of a function, while the Heine's definition is convenient to disprove the existence of limit.

The example given demonstrates that the concept presentation variability is closely related to the function of pragmatism, i.e. its applicability to solve one problem or another. Thereby, along with the semantics of a concept, i.e. 
with the meaning contents of a concept, one should take into consideration the pragmatism of a concept, i.e. its applicability as a method for a presentation of other concepts (language aspect) or a method for proof / disproof (instrumental aspect).

Another example illustrating semantic and pragmatic aspects of the concept is the concept of the 'Truth table for logical functions'. As the definition the concept falls into semantics, meanwhile its application as an instrument to prove equivalence in algebra of logics bears a pragmatic character.

6. Homomorphism.Homomorphism is seen as "coarsening". of concepts. A typical situation of a homomorphism arises in the simulation of objects. The first step in this process is determination the most essential factors that characterize the object, i.e. as if it is "coarsened". When applying this cognitive structure we always know exactly what we do neglected, how we obtain the result (at what expense) and what dangers lie in wait for us in connection with the application of homomorphic models rather than isomorphic ones.

7. Concept inclusion.We say that a concept A includes a concept $B$ if the application sphere of the concept A includes the application sphere of the concept B although the concept Acan be subordinated to the concept B logically.

We don't consider this invariant here, because for now it is fixed by us only in the mathematical disciplines. Interested parties can see examples of its application in[5].

As mentioned above, J. Piaget's discovery of the cognitive invariants ('classification' and 'seriation') is the basis to construct diagnose of children's thinking. In terms of the cognitive invariants specified by us we consider them to be appropriate for diagnostics of the level of thinking development the older age groups, in particular upperclassmen and students. We see an indirect confirmation of the thesis in the implicit use of cognitive invariants as isomorphism and language presentations in the researches of J. Raven [8], R. Sternberg [9], A. Brushlinsky [10]. In [11] this has been shown.

An important feature of metacognitive invariants is that mastering of them does not increase the loading of students but rather allows you to build teaching methodology that makes the learning more effective.Moving on to a discussion of their role in the method of constructing learning we note first of all that the logical subordination, the priority of which is emphasized in building any course, really is a partial order rather than a full order. Already this fact often leads to methodical conflicts connected with the required linear account of the course.The presentation of the logical dependence between various elements of the course contents (concepts, facts, statements, etc.) looks like an oriented graph, the vertices being elements of the contents and the edges indicate the logical consequence.The distance measurement procedure on the graph is naturally defined as the shortest path between two vertices.Thus arises the concept of the logical proximity of elements of the content.

However, in terms of teaching methods the logical proximity of elements in the content is not the only kind of proximity. There exist other links arising between concepts from the analogy, similarity methods used, etc.These concepts are connected not because of the structure of logical consequence but in view of other factors which are often more important to the didactic and methodical perspectives. The metacognitive invariants discussed above are to be attributed to these factors since they reflect the nature of the relationship between the various concepts bringing them closer in the process of learning. Formalizing of the intuitive proximity understanding gives an additional topological structure on the graph of the logical relationships between concepts. The trajectory of the material presentation, taking into account the conceptual proximity, enables to 'joint' logically remote parts of the course to gain a better understanding of integrative character its separate elements. The approach facilitates the students to understand the integrity of the course and for teachers it demonstrates the depth relationships of separate elements of the discipline. The understanding of relations between concepts develops the students thinking and ensures acquisition of knowledge at the content level rather than formal one.

That's just one example from the course of 'Discrete Mathematics' where invariant of 'isomorphism' was used.

It should be noted that students successfully acquire set-theoretic operations and their properties. If the elements of the set $A$ are considered to be the points of the circle, for example, the students looking at the picture (Venn diagram or Euler diagrams) understand that self-intersection and self-union of the set $A$ equals to the set $A$, i.e. $A \cap A=A$ and $A \cup A=A$.

Meanwhile, similar identity of logics algebra is more difficult for the students to acquire due to their false identification with arithmetic operations.Very few of them immediately understand that if $x$ is a logical rather than algebraic variable than logical multiplication (conjunction) and logical addition (disjunction) satisfy identities $x \cdot x=x$ and $x \vee x=x$, other than $x^{2}$ and $2 x$, respectively like in algebra.

The juxtaposition the set $A$ with the logical variable $x$ and the set-theoretical operations of 'intersection' and 'union' with the operations of 'conjunction' and 'disjunction' gives the isomorphism of the concepts and operations.

To be able to assess the degree of optimization of teaching using metacognitive invariants, we proposed a mathematical model $[5,12]$, based on metric ratios of the graph of the content enriched by the system of the concept links. By the example of the course of 'Discrete Mathematics' it has been shown that the construction of the educational trajectory with the using of conceptual connections can save up to $24.1 \%$ of the study time. As a result, the students have an opportunity to a deeper and more thorough exploration of this course. It goes without saying that there are other factors coming into force when constructing the actual teaching. So achieve the parameters' value calculated according to our model should not be expected. However, the effect has been observed, as evidenced by the results of the pedagogical experiment 
conducted at teaching the course 'Discrete Mathematics'.

\section{Results}

The pedagogical experiment employing the instruction methods based on the metacognitive invariants has been undertaken in four groups of students. The students of the one academic group were taught by conventional methods. The average score level of students in the experimental groups was $4 \%-8 \%$ higher than that of the students in the control groups. Vividly effect of change in teaching methods observed when comparing the examination scores: in the control group the proportion of high rates was $58 \%$ and in the experimental groups it was from $69 \%$ to $77 \%$.

\section{Discussion and Conclusions}

The following conclusions are drawn out of the experiment undertaken.

1. The experimental groups' students have had a higher level of knowledge.

2. A steady rising of the average score for the subject indicates a higher quality acquisition of the entire subject.

3. The application of the metacognitive invariants mechanism ensures a steady rise of the higher scores over $10 \%$ in the experimental groups vs. those of in the control groups.

\section{REFERENCES}

[1] Raven J. Competence in Modern Society: Its Identification, Development and Release. Unionville, New York: Royal
Fireworks Press, 1997.

[2] Hutmacher Walo. Key Competencies for Europe. Report of the Symposium Berne, Switzerland 27-30 March, 1996. Council for Cultural Co-operation (CDCC) a Secondary Education for Europe. Strasburg, 1997.

[3] Aronov A.M., Znamenskaya O.V. On the concept of "Mathematic Competence" (Russian). Bull. Moscow University, Series Pedagogical Education, № 4, 31-43, 2010.

[4] Piaget J. Logic and Psychology, Manchester, University Press, 1953.

[5] Gein A.G., Nekrasov V.P. Simulation of Concept Links Development (Russian), Yekaterinburg, UTICCS, 2011.

[6] Mochalov A.N., Gein A.G., Nekrasov V.P. Metasubject Model in Science of Law (Russian), Bull. Ural Institute of Economics, Management and Law, № 1, 83-89, 2012.

[7] Polya G. How to Solve It, Princeton University Press, Princeton, New Jersey, 1973

[8] Raven J.C. Guide to the Progressive Matrices, Lewis, London, 1960.

[9] Sternberg R.J. The concept of "giftedness": A pentagonal implicit theory, The origins and development of high ability. Wiley, Chichester, (Ciba Foundation Symposium), 5-16, 1993.

[10] Brushlinsky A.V. Thinking and forecasting (Russian), Mysl', Moscow, 1979.

[11] Gein A.G., Nekrasov V.P. Methasubject constructs as formation factors of higher school undergraduates' cognitive competence (Russian), Bull. Moscow University, Series Pedagogical Education. № 4, 43-61, 2012.

[12] Gein A.G., Nekrasov V.P. On Quantitative Estimation of Mathematics Course Didactic Saturation (Russian), Bull. Viatskiy State University of Arts, № 4 (3), 63-67, 2010. 